\title{
An Evolutionary Approach to Modeling Radial Brightness Distributions in Elliptical Galaxies
}

\author{
$\mathrm{Jin}^{\mathrm{Li}}{ }^{1}$, Xin $\mathrm{Yao}^{1}$, Colin Frayn ${ }^{1}$ \\ Habib G. Khosroshahi ${ }^{2}$, Somak Raychaudhury ${ }^{2}$ \\ ${ }^{1}$ The Centre of Excellence for Research \\ in Computational Intelligence and Applications (CERCIA), School of Computer Science, \\ The University of Birmingham, Edgbaston, Birmingham B15 2TT, UK \\ \{J.Li, X.Yao, C.M.Frayn\}@cercia.ac.uk \\ http://www.cercia.ac.uk/index.html \\ ${ }^{2}$ Astrophysics and Space Research Group, School of Physics and Astronomy \\ The University of Birmingham, Edgbaston, Birmingham, B15 2TT, UK \\ \{habib, somak\}@star.sr.bham.ac.uk
}

\begin{abstract}
A reasonably good description of the luminosity profiles of galaxies is needed as it serves as a guide towards understanding the process of galaxy formation and evolution. To obtain a radial brightness profile model of a galaxy, the way varies both in terms of the exact mathematical form of the function used and in terms of the algorithm used for parameters fitting for the function given. Traditionally, one builds such a model by means of fitting parameters for a functional form assumed beforehand. As a result, such a model depends crucially on the assumed functional form. In this paper we propose an approach that enables one to build profile models from data directly without assuming a functional form in advance by using evolutionary computation. This evolutionary approach consists of two major steps that serve two goals. The first step applies the technique of genetic programming with the aim of finding a promising functional form, whereas the second step takes advantage of the power of evolutionary programming with the aim of fitting parameters for functional forms found at the first step. The proposed evolutionary approach has been applied to modeling 18 elliptical galaxies profiles and its preliminary results are reported in this paper.
\end{abstract}

\section{Introduction}

The basic building block of the universe is the galaxy. Nevertheless, even though the physics of the formation and evolution of individual stars is well understood, how galaxies form and evolve in various environments is not very well understood. In optical images, galaxies have a wide range of shapes and sizes. The appearance of a galaxy is expected to be related to its formation evolutionary history, and thus the morphological classification of galaxies is considered to be an important exercise in astrophysics (van den Bergh 1998).

Most regular galaxies can be divided into two types, namely, ellipticals and spirals. Elliptical galaxies appear smooth and structureless and have elliptical isophotes. In contrast, spiralarms. galaxies comprise of a central brightness condensation resembling an elliptical, called the bulge, and a thin disk, which contains obvious spiral features. To develop a deeper understanding of the evolution of galaxies, it is 
important to quantify the morphological features as a first stage in an attempt to link them to the physics of their formation .

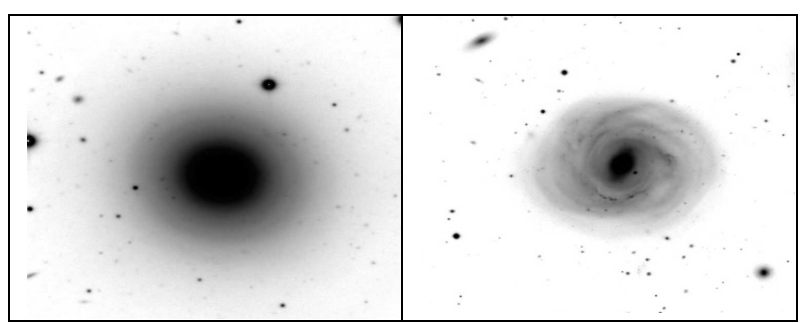

Fig. 1. Monochromatic (negative) images of a typical elliptical (left) and spiral (right) galaxy.

For this purpose it is necessary to express the structural features of a galaxy in terms of a small set of parameters. One way of doing this is to obtain a radial brightness profile of the galaxy, i.e., how the galaxy surface brightness varies as a function of radial distance from its center. Substantial work has been done towards finding better analytic fitting functions for radial brightness profiles ${ }^{1}$ of galaxies. These include work by Hubble (1930), de Vaucouleurs (1948), King (1962), Sersic (1968) and Kormendy (1977). The most commonly used fitting functions for elliptical galaxy profiles are the Hubble's law given by

$$
I(r)=I_{0} /(r / a+1)^{2}
$$

and the de Vaucouleurs $\mathrm{r}^{1 / 4}$ law given by

$$
I(r)=I_{e} \exp \left\{-3.33\left[\left(r / r_{e}\right)^{1 / 4}-1\right]\right\}
$$

Each function has two parameters, a brightness scale $I_{0}$ or $I_{e}$ and a size scale $a$ or $r_{e} . I_{0}$ is the surface brightness at $r=0$, i.e. the central surface brightness. The scale length $a$ is the distance at which the surface brightness falls to a quarter of its central value. In constrast, $r_{e}$, known as the effective radius, contains half of the total light of the galaxy, and $I_{e}$ is the brightness at $r_{e}$. The two laws give a reasonably good description of the luminosity profiles of elliptical galaxies and the bulges of some types of spiral galaxies. However, they do not describe well these profiles beyond certain bounds of $r$ or the bulges of spiral galaxies with pronounced spiral features. For the latter, a popular model is an exponential form

$$
I(r)=5.36 I_{e} \exp \left[-1.68\left(r / r_{e}\right)\right] .
$$

Neither (2) nor (3) have been formally linked to a physical model of galaxy formation, and remain mere empirical fits to observed profiles. Hubble's model (1) is not a very good fit to a wide range of observed ellipticals, but is related to possible density profiles of self-gravitating isothermal spheres of gas. It would be desirable to have a single model that would describe all elliptical galaxies with a variation of parameters, in particular if this model could be linked with the physics of collapsing gas clouds and the formation of self-gravitating ellipsoidal systems.

1 The radial surface brightness profile will hereafter be simply referred to as the "profile". 
On the other hand, spiral galaxies are dominated by a disk which seems to be reasonably described by a simple exponential brightness profile (Freeman 1970)

$$
I(r)=I_{0} \exp (-r / h)
$$

where $I_{0}$ is the central surface brightness and $h$ represents the scale-length of the disk.

There has been a lot of effort in the literature to find appropriate mathematical functions to describe a wide range of profiles of various components of spiral and elliptical galaxies, and to find plausible values for parameters that would fit observed profiles. Both tasks are non-trivial. The usual approach is to postulate a mathematical model comprising of common mathematical functions, then to apply fitting algorithms to find suitable parameters for these functions. The parameter fitting algorithm usually adopted is the non-linear reduced $\chi^{2}$ minimization given by

$$
\chi^{2}=\frac{1}{v} \sum_{i} \frac{\left[I_{m o d e l}(i)-I_{o b s}(i)\right]^{2}}{\delta^{2}}
$$

where $I_{o b s}(i)$ is the individual observed profile value, $I_{\text {model }}(i)$ is the value calculated from the fitting function, $v$ is the number of degrees of freedom, and $\delta$ is the standard deviation of the data. One disadvantage of non-linear minimization algorithms is their sensitivity to the initial values provided. Unreasonable initial values could cause the fitting program to trap in a local minimum. (see Andredakis \& Sanders 1994, de Jong R.S. 1996 and Wadadekar et al 1999).

As more and more galaxy images have become available, the popular models seem to be suitable for an increasingly smaller fraction of observed galaxies. With the imminent prospect of photometry of millions of galaxies becoming publicly available through the Virtual Observatory project (www.ivoa.net), it is crucial to find reasonably good generic mathematical models that would describe a much larger number of galaxies with variation in model parameters.

In our approach presented here, the technique of genetic programming (GP) (Koza, 1992) plays the role as the first step to find alternative promising forms. The second step in our approach aims at fitting parameters in the function thus derived. To achieve the second goal, we take the evolutionary programming (EP) (Fogel, 1966; Fogel, 1991) technique rather than non-linear reduced $\chi^{2}$ minimization algorithms. The main reason for this is that EP has successfully worked for many numerical and combinatorial optimization problems in recent years (Yao 1996, 1999). With its population of solutions, EP makes it easier to escape local minima and is more likely to find global minima.

The structure of the paper is as follows. Section 2 describes the sample of 18 radial brightness profiles of elliptical galaxies that we use in this study. Section 3 presents details of the evolutionary approach that involves genetic programming and evolutionary programming. In Section 4 , we present our experiments on the application of our evolutionary approach to modeling 18 profiles and also report preliminary results. We draw conclusions and discuss our further work in Section 5.

\section{The distribution of light in Elliptical Galaxies}




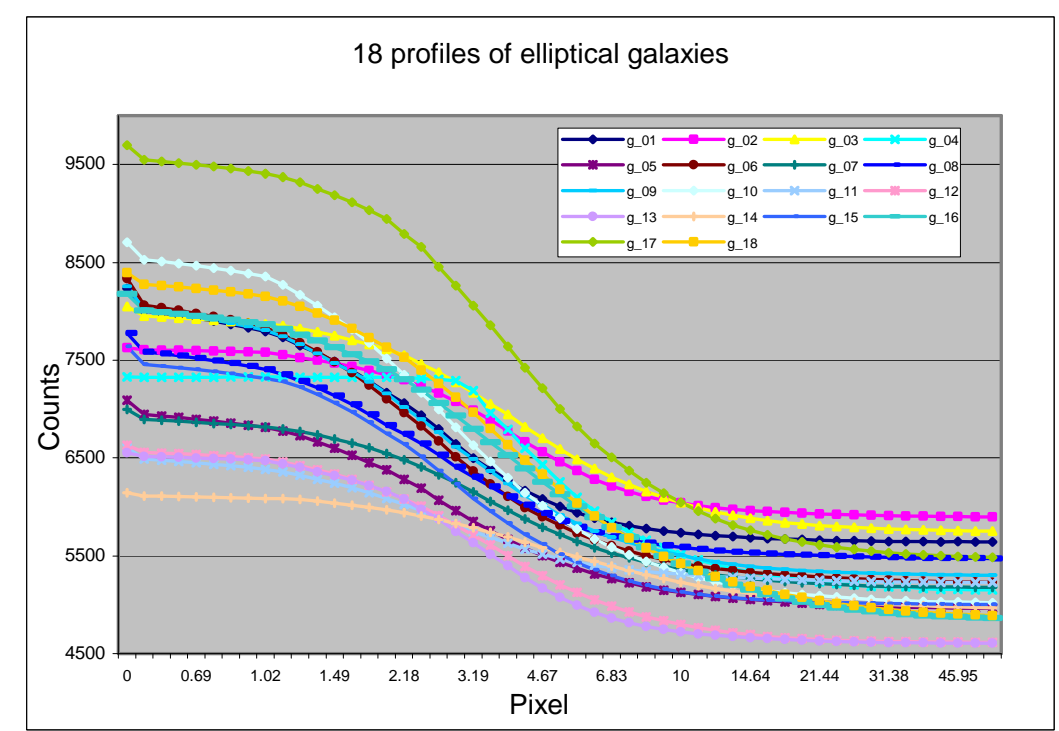

Fig. 2. The brightness intensity distribution of 18 elliptical galaxies

The data chosen for this study is a set of 18 elliptical galaxies in the Coma cluster observed in near-infrared band (central wavelength $2.2 \mu \mathrm{m}$; for further description of the observations see Khosroshahi et al 2000a). Elliptical galaxies are chosen for this study because of their smooth brightness distribution compared to the more complicated morphologies of spiral galaxies. They are also chosen from the same cluster of galaxies to enable us to eliminate the signature of different environments on the surface brightness of the sample galaxies. Elliptical galaxies historically have been characterized by the de Vaucouleurs $\left(\mathrm{r}^{1 / 4}\right)$ profile, which is an empirical fit, not yet shown to emerge from theoretical or semi-analytic scenarios of galaxy formation. Careful observations reveal a far greater variety of profiles, leading, among others, Khosroshahi et al $(2000 \mathrm{a}, 2000 \mathrm{~b})$ to advocate the use of generalized models that allow for shallower or steeper profiles to elliptical galaxies and bulges of disk galaxies.

In this exercise, one dimensional radial profiles have been extracted from fitting elliptical contours of the same brightness (isophotes) to the images of the galaxies, and computing the average brightness as a function of radius along the major axis of the ellipse at various values of the radial distance $r$. Each profile has 50 data points. The sky background is highly variable at near-infrared wavelengths. Therefore the local background is highly variable (Fig 2).

\section{The Evolutionary Approach}

Our evolutionary approach consists of two major steps, each step having its distinct target. First we aim to seek promising mathematical functions using genetic programming (Koza, 1992). With the function generated, a simple generalization process for the functional form is necessary. We generalize the form in the way of replacing a few numerical values by a few parameter variables. 
The second step is aimed at fitting parameters in the function formula thus found, using evolutionary programming techniques. The details of both algorithms used in our approach are described below.

\subsection{Genetic Programming}

Genetic programming (GP) is a class of evolutionary computation inspired by Darwin's evolution theory. It operates iteratively evolving a population of individuals. On each iteration, all individuals are evaluated in terms of the fitness function. A new population is then generated by probabilistically selecting the most fit individuals from the current population. Some members in the new population are carried forward from the last generation population intact via reproduction operation. The rest are generated by applying genetic operators: crossover or mutation. Such a process continues until sufficiently fit individuals are found.

GP has been applied to a variety of fields (Koza 1994). One important application is symbolic regression (or function identification). Problems of symbolic regression require finding a function, in symbolic form, which fits a given limited sampling of data points. One individual in GP is an executable tree structure/parse tree, which can be interpreted as a mathematical expression. GP is capable of generating mathematical formulas which approximate many or even whole data points among a set of data sample given. In addition, because GP algorithm is a stochastic process, it is able to generate a variety of function forms when modeling even on the same data. These facts make GP a strong candidate for finding alternative promising forms.

For simplicity, the detail of GP algorithms is not presented here. For readers interested, please refer to the book (Koza 1992). In the case of GP here, we take a function set, $F=\{+,-, *, /$, exp, log, sin, $\cos \}$ and a terminal set, $T=\{\mathrm{r}, \mathfrak{R}\}$ where $\mathrm{r}$ is the variable radius and $\Re$ is a random float-point value between -10.0 and 10.0. Theoretically, all profile models (function 1-4) introduced in Section 1 could be potentially generated by GP. Examples of individual expressions are illustrated in Figure 3.
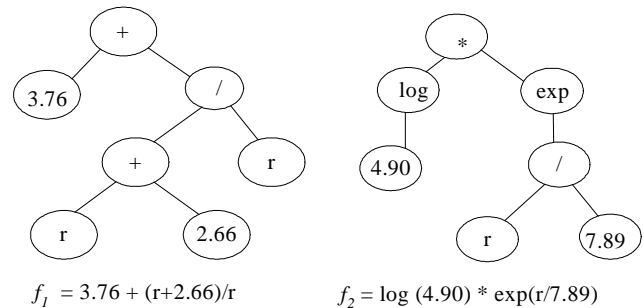

$f_{2}=\log (4.90) * \exp (\mathrm{r} / 7.89)$

Fig. 3. Examples of mathematical formula forms created by GP

The fitness function is one of the most important components in GP. Here it determines how well an individual expression fits an observational galaxy profile. The overall fitness function used here for GP consists of two items. We take hits as the first part of the fitness function. The 'hits measure' here counts the number of fitness data points for which the numerical value returned by the model expression lies within a small tolerance (the 'hits criterion') of the observed intensity value. For example, the hits criterion taken in this paper is 0.005 for GP. Selecting an appropriate value for the hit criterion seems a bit tricky here. In general, a higher hit criterion could fail to 
guide GP to find good shapes of functions that fit profiles properly. In contrast, a lower hit criterion does allow GP to find a good formula which could possibly over-fit a particular profile, but lack generality to match other profiles. This is also known as the over-fitting problem in machine learning domain. The aim of using GP is to find promising functional forms that potentially have capability for describing as many profiles as possible. Therefore, in our approach, taking a higher hit criterion for GP is preferable as long as the maximum number of hits (i.e. 50 in this study) is achievable in terms of the results on 18 galaxies profiles.

In addition, we add a second item into the fitness function to penalize any complex mathematical expression because a simple functional form is almost always preferable. The complexity of an expression is measured by the length of the expression, i.e. the number of nodes within the expression. The extent to which an expression should be penalized is changeable by a weight $w$. In summary, the fitness function that we use is given by

$$
f_{\text {fitness function }}=\text { Hits }-w * \text { the length of the expression. }
$$

It is worth noting that the second item in the fitness function is also essential. Without it, our GP tends to generate rather complicated mathematical functional forms, which are difficult to generalize.

The criteria for terminating a GP run are either the maximum run time allowed or the maximum of generation that we set, whichever reached first.

\subsection{Evolutionary Programming}

GP is good at finding a function or a symbolic form. However, it lacks mechanism to tune parameters in the form. This is partly because existing genetic operations, such as crossover or mutation, affect only the structure of the trees, not the composition of the nodes. The numeric constants in nodes thus cannot benefit much from them.

Like GP, evolutionary programming is one class of evolutionary computation (Fogel, 1966). Unlike GP, EP merely uses mutation as its genetic operator and has only two major steps: 1) mutate the solutions in the current population; 2) select the next generation from the mutated and the current solutions. By means of its population of solutions, EP makes it easier to escape local minima and is more likely to find global minima. The effectiveness of EP has been demonstrated in solving many numerical and combinatorial optimization problems in recent years (Yao 1996).

In this study, the EP algorithm is similar to one we used in (Yao, 1999), except that we do not apply self-adaptive mutation, which a classical EP usually takes. It is simply because the simple mutation strategy works fairly well on the problems being solved here. We use the Cauchy mutation rather than traditional Gaussian mutation as our previous work has demonstrated that the former performs much better than the latter (Yao, 1999). The fitness function we take is merely the hits, the first item of the fitness function for GP. The termination criterion taken here is that the maximum generation has reached.

\section{Experiments and Results}

The experiments in this study were carried out as follows. We first ran the GP system on each of 18 galaxy profile datasets. It is common practice to fit in the logarithmic 
(magnitude) regime when decomposing 1D profiles. We adopt this approach and let GP run to fit the model function forms to data points in the logarithmic regime for each profile. The parameter settings for running GP are listed in the Table 1.

Table 1. Parameter settings used in our GP for experiments

\begin{tabular}{|c|c|}
\hline Target & $\begin{array}{l}\text { To find a regression mathematical function } \\
\text { based on the galaxy profile given }\end{array}$ \\
\hline 'Terminals & $R, \mathfrak{K}=[-10,10]$ \\
\hline Non-terminals & $+,-, *, /, \exp , \sin , \cos , \log$ \\
\hline Crossover rate & 0.9 \\
\hline Mutation rate & 0.01 \\
\hline Population size & 6,000 \\
\hline Maximum no. of generations & 100 \\
\hline Termination criterion & $\begin{array}{l}\text { Generation limit or Time limit, whichever } \\
\text { reached first }\end{array}$ \\
\hline Selection strategy & Tournament selection, Size $=6$ \\
\hline Max depth of individual expressions & 17 \\
\hline Hit criterion & 0.005 \\
\hline Fitness criterion & Hits $-0.01 *$ the length of the expression \\
\hline Max depth of initial individual expressions & 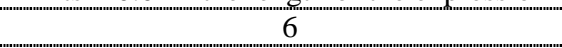 \\
\hline "Maximum run time (hours) & 6 \\
\hline
\end{tabular}

One profile model is created after each GP run. 18 different profile models with different mathematical forms are generated in total. They seem different in their shapes of tree structure. Therefore, finding similarity between those forms generated is needed in order to find a more generic model form.

We simplify a tree-structured function form in the way of cutting each possible subtree and replacing it by one single numerical node if the ultimate return value of the sub-tree is a numerical value. After the simplification, the form has to be generalized in the way of replacing a few numerical values by variables (e.g. $a, b$, and $c$ ) respectively.

After the generalization process, there emerge a few forms with parameters that are relatively simple. Among these forms we only select the following two simplest function forms $f_{\mathrm{g} 1}$ and $f_{\mathrm{g} 2}$ for further investigation.

$$
\begin{gathered}
f_{\mathrm{g} 1}=a+b /(c+r) \\
f_{\mathrm{g} 2}=a^{\prime}+b^{\prime} /\left(b^{\prime}+c^{\prime} r^{2}\right)
\end{gathered}
$$

Each of the two forms involves three parameters i.e. $a, b$, and $c$ for $f_{g 1} ; a^{\prime}, b^{\prime}$ and $c$ ' for $f_{\mathrm{g} 2}$, and one radius $r$. Now, we use EP to seek proper values for three parameters within the both functions respectively to fit each galaxy profile in the logarithmic regime. The parameter settings for running EP are listed in the Table 2.

For brevity, results of fitting parameters found by EP for $f_{\mathrm{g} 1}$ and for $f_{\mathrm{g} 2}$ are both listed in Table 3. 
Table 2. Parameters used in our EP for experiments

\begin{tabular}{c|c}
\hline Target & To find proper parameters \\
\hline Mutation rate & 0.9 \\
\hline Population size & 10,000 \\
\hline Maximum no. of generations & 150 \\
\hline Termination criterion & Maximum generation reach \\
\hline Selection strategy & Tournament selection, Size $=6$ \\
\hline Fitness criterion & Hits (maximum hit $=50)$ \\
\hline Hit criterion & 0.005 \\
\hline
\end{tabular}

Table 3. Fitting parameters found by EP for functions $f_{\mathrm{g} 1}$ and $f_{\mathrm{g} 2}$ using 0.005 as a hit criterion

\begin{tabular}{c|c|c|c|c|c|c}
\hline \multirow{2}{*}{ Profiles } & \multicolumn{3}{|c|}{$f_{\mathrm{g} 1}$} & \multicolumn{2}{c}{$f_{\mathrm{g} 2}$} \\
\cline { 2 - 7 } & $a$ & $b$ & $c$ & $a^{\prime}$ & $b^{\prime}$ & $c^{\prime}$ \\
\hline 1 & 3.7303 & 0.4272 & 1.7655 & 3.7681 & 0.1427 & 0.2193 \\
\hline 2 & 3.7444 & 0.7167 & 4.8130 & 3.7886 & 0.0999 & 0.1485 \\
\hline 3 & 3.7308 & 1.1017 & 6.0725 & 3.7700 & 0.1349 & 0.0943 \\
\hline 4 & 3.6701 & 1.4376 & 6.2036 & 3.7276 & 0.1579 & 0.0386 \\
\hline 5 & 3.6741 & 0.6300 & 3.0043 & 3.7073 & 0.1222 & 0.1078 \\
\hline 6 & 3.6897 & 0.6138 & 2.1494 & 3.7272 & 0.1712 & 0.0872 \\
\hline 7 & 3.6878 & 0.7763 & 4.8547 & 3.7316 & 0.1125 & 0.0663 \\
\hline 8 & 3.7217 & 0.4731 & 2.3636 & 3.7547 & 0.1259 & 0.1589 \\
\hline 9 & 3.7074 & 0.6060 & 2.4258 & 3.7347 & 0.1908 & 0.1721 \\
\hline 10 & 3.6695 & 0.8550 & 2.5385 & 3.7093 & 0.2387 & 0.1462 \\
\hline 11 & 3.6893 & 0.7154 & 5.7652 & 3.7253 & 0.0910 & 0.0884 \\
\hline 12 & 3.6373 & 0.8105 & 3.7815 & 3.6796 & 0.1565 & 0.1438 \\
\hline 13 & 3.6382 & 0.6320 & 2.7736 & 3.6808 & 0.1408 & 0.1257 \\
\hline 14 & 3.6987 & 0.2636 & 2.1155 & 3.7096 & 0.0959 & 0.1510 \\
\hline 15 & 3.6712 & 0.6221 & 2.3377 & 3.7002 & 0.1973 & 0.1582 \\
\hline 16 & 3.6804 & 0.8437 & 2.9601 & 3.6999 & 0.2267 & 0.0779 \\
\hline 17 & 3.6960 & 1.4928 & 4.4057 & 3.7479 & 0.2550 & 0.0623 \\
\hline 18 & 3.6515 & 1.4413 & 4.7698 & 3.7065 & 0.2213 & 0.0741 \\
\hline
\end{tabular}

Several points are worth indicating here. First of all, EP algorithms almost always achieve the maximum hits: 50 (the number of data points for each profile), with the above EP parameter settings while running based on either function forms for each galaxy profile. This is partly attributed to a large population of solutions. Secondly, unlike non-linear fitting algorithms, EP is not sensitive to the initial values provided at all. Throughout our experiments we keep the initial values for those parameters (i.e. $a$, $b$, and $c ; a^{\prime}, b^{\prime}$ and $c^{\prime}$ ), which do not need to be tuned in order to avoid failure of a run. This is attributed to the mutation in EP. Thirdly, the hit criterion is one of the most important parameter settings in EP because it determines how well the resulting models fit the empirical galaxy profile. The smaller the value of the hit criterion is, the better fit the resulting model could be. This will be further discussed shortly.

To evaluate the accuracy and overall success of a model fit, we use statistical measure, the reduced $\chi^{2}$, given in (5). In general, the resultant $\chi^{2}$ above 2 means that the model does not seem to describe the empirical galaxy profile very well. Otherwise, the model is a good description for the brightness distribution of a galaxy. We calculate the reduced $\chi^{2}$ for each galaxy based on both functions respectively. Both results are shown under the columns with hit criterion $=0.005$ in Table 4 . 
Table 4. The reduced $\chi^{2}$ for 18 galaxies models based on two function $f_{\mathrm{g} 1}$ and $f_{\mathrm{g} 2}$ using two hit criteria

\begin{tabular}{c|c|c|c|c}
\hline \multirow{2}{*}{ Profiles } & \multicolumn{2}{|c|}{$\chi^{2}$ for $f_{\mathrm{g} 1}$} & \multicolumn{2}{c}{$\chi^{2}$ for $f_{\mathrm{g} 2}$} \\
\cline { 2 - 5 } & $\begin{array}{c}\text { Using hit crite- } \\
\text { ria }=0.005\end{array}$ & $\begin{array}{c}\text { Using hit crite- } \\
\text { ria }=0.002\end{array}$ & $\begin{array}{c}\text { Using hit cri- } \\
\text { teria }=0.005\end{array}$ & $\begin{array}{c}\text { Using hit crite- } \\
\text { ria }=0.002\end{array}$ \\
\hline 1 & 7.4460 & 7.8961 & 11.4012 & $\mathbf{1 . 1 1 4 1}$ \\
\hline 2 & 9.1809 & 6.2876 & 12.8405 & $\mathbf{1 . 8 6 3 3}$ \\
\hline 3 & 8.7796 & 5.0199 & 6.3452 & $\mathbf{0 . 7 1 1 3}$ \\
\hline 4 & 6.8782 & 6.8221 & 2.8035 & 2.8385 \\
\hline 5 & 4.1948 & 3.8105 & 5.7141 & $\mathbf{0 . 8 6 8 5}$ \\
\hline 6 & 3.1867 & 2.7282 & 3.2442 & $\mathbf{0 . 7 1 9 4}$ \\
\hline 7 & 9.7797 & 4.8050 & 17.9798 & $\mathbf{0 . 9 2 8 9}$ \\
\hline 8 & 6.8756 & 4.9609 & 11.0505 & $\mathbf{0 . 1 3 5 3}$ \\
\hline 9 & 5.8373 & 5.0018 & 3.4484 & $\mathbf{1 . 0 3 9 0}$ \\
\hline 10 & 2.8263 & 2.8807 & $\mathbf{1 . 9 0 7 8}$ & $\mathbf{0 . 3 3 1 8}$ \\
\hline 11 & 34.0802 & 8.2256 & 25.2975 & 7.9733 \\
\hline 12 & 5.8477 & 5.0861 & 7.9886 & $\mathbf{1 . 5 1 5 0}$ \\
\hline 13 & 7.6026 & 7.6528 & 10.6681 & $\mathbf{1 . 2 6 1 5}$ \\
\hline 14 & 9.1534 & $\mathbf{1 . 8 2 9 9}$ & 13.8083 & $\mathbf{1 . 2 8 9 6}$ \\
\hline 15 & 5.9548 & 5.8170 & 2.1233 & $\mathbf{1 . 2 0 6 2}$ \\
\hline 16 & 4.8048 & $\mathbf{1 . 9 8 7 1 9}$ & 3.7705 & $\mathbf{0 . 6 7 5 9}$ \\
\hline 17 & 2.9778 & 3.0106 & $\mathbf{1 . 3 4 1 8}$ & $\mathbf{0 . 3 1 3 8}$ \\
\hline 18 & $\mathbf{1 . 2 1 6 1}$ & $\mathbf{1 . 0 2 3 3}$ & $\mathbf{0 . 8 0 3 1}$ & $\mathbf{0 . 0 7 9 6}$ \\
\hline
\end{tabular}

According to the value of the reduced $\chi^{2}$, only one model based on $f_{\mathrm{g} 1}$ fits the observational profile very well (i.e. galaxy 18 ; its $\chi^{2}=1.2161<2$ ), whereas three models based on $f_{g 2}$ perform well for galaxy 10 , galaxy 17 and galaxy 18 as each $\chi^{2}$ is less than 2 . We may argue that $f_{g 2}$ is a better model than $f_{\mathrm{g} 1}$ in terms of the total number of good model fittings. Apart from this fact, the results do not seem promising at all. The poor performance is partly due to the higher hit criterion we set in EP.

As mentioned earlier, the hit criterion in EP determines how closely a resulting model fits an empirical galaxy profile. A lower hit criterion potentially guides EP to find appropriate values of parameters which can match empirical models better. The poor results here imply that a lower hit criterion is necessary to make models better.

We then carry out a set of experiments using a smaller hit criterion 0.002 . All other parameter settings for EP are the same except for the maximum number of generations. We double the maximum number of generation to 300 as the termination criterion for EP. For simplicity, results of parameter values found by $\mathrm{EP}$ for $f_{\mathrm{g} 1}$ (i.e. $a, b$, and $c$ ) and $f_{\mathrm{g} 2}$ (i.e. $a^{\prime}, b^{\prime}$ and $c^{\prime}$ ) are not shown in this paper. We only list the results of the reduced $\chi^{2}$ based on both function models to see how good those resultant models are. The results are shown under the column with the hit criterion $=0.002$ in Table 4 .

With the smaller hit criterion, only three models based on $f_{\mathrm{g} 1}$ have been found to be fairly good because their $\chi^{2}$ s calculated are all less than 2 . In contrast, based on $f_{\mathrm{g} 2}, 16$ models among the total 18 are good descriptions for corresponding galaxy profiles except for two models, i.e. the model 4 for galaxy 4 and the model 11 for galaxy 11 . The $\chi^{2}$ s of model 4 and model 11 are 2.8385 and 7.7933 respectively.

While comparing the results by using 0.005 as a hit criterion, we note that more good fitting models can be found by using 0.002 for both $f_{\mathrm{g} 1}$ and $f_{\mathrm{g} 2}$ in terms of the standard of the reduced $\chi^{2}$. The number of good models found increases from 1 to 3 
for $f_{\mathrm{g} 1}$ and from 3 to 16 for $f_{\mathrm{g} 2}$. In terms of the total number of good fitting galaxies found here, we argue again that $f_{g 2}$ is a more promising mathematical form compared with $f_{\mathrm{g} 1}$ to describe 18 galaxy profiles studied here.

\section{Conclusions and Future Work}

In the study of galaxy formation and evolution, it is important to find functional forms that best describe the distribution of starlight in galaxy images. Limitations of traditional approaches are: a) an exact mathematical function form must be given before applying any fitting algorithms; b) the commonly used fitting algorithm like non-linear reduced $\chi^{2}$ tends to be sensitive to initial values provided and more likely culminates in wrong local minima, resulting in unsatisfactory fits.

An evolutionary approach has been proposed in this paper. It attempts to overcome the above weakness of traditional methods. The approach takes two major steps to achieve distinct goals. The first step uses a genetic programming technique to find promising mathematical functional forms based on observed radial profiles of elliptical galaxies. The second step applies evolutionary programming aimed at finding appropriate values for the parameters within the form found. Between the two steps, the process of generalization is required to make the overall approach succeed. The main novelty of the approach lies in the fact that the whole procedure of modelling profiles is a data-driven process without assuming a functional form beforehand. This bottom up process is particularly useful when one faces a large number of galaxy profiles without any prior knowledge of them. It allows one to find a good functional form first and then to fit parameters for the functions in order to build reasonably good galaxy profile models.

The approach has been demonstrated on 18 galaxy profiles given. Two major different mathematical function forms are found at its first step. Through a generalization process, three parameters are introduced into each function. Parameter fittings by EP using two different hit criteria have been carried out at its second step. Experimental results demonstrate that a good mathematical form plays a more important part in finding good descriptions of galaxy profiles. On the other hand, a smaller hit criterion is preferable to use in order to guide EP to achieve better models.

In this paper, we have conducted a preliminary study to apply our proposed evolutionary approach to modelling a number of galaxy profiles. Though initial results are promising, the effectiveness of our approach needs further investigation. In particular, we would like to test the effectiveness of the evolutionary approach to a great extent. The number of galaxy profile samples would be increased in our future study. In the meantime, other types of galaxies should also be considered in our research. As twodimensional decomposition techniques are becoming more promising for modeling galaxy profiles (Wadadekar et al, 1999; Khosroshahi et al, 2000b), exploring the possibility of combining the evolutionary approach with two-dimensional decomposition techniques certainly merits further investigation. 


\section{References}

Andredakis, Y. C., Sanders, R. H. (1994). Exponential bulges in late-type spirals: an improved description of the light distribution, Monthly Notices of the Royal Astronomical Society (MNRAS), Vol. 267, No. 2, 283-296.

De Jong, R.S. (1996), Near-IR photometry of 86 galaxies. II. Astronomy \& Astrophysics Supplement Series, 118, 557-573.

De Vaucouleurs G. (1948). Recherches sur les Nebuleuses Extragalactiques. Annales d'Astrophysique, Vol. 11, 247.

Fogel, D. B. (1991). System Identification Through Simulated Evolution: A Machine Learning Approach to Modeling. Needham Heights, MA: Ginn.

Fogel, L. J., Owens, A. J. and Walsh, M. J. (1966). Artificial Intelligence Through Simulated Evolution. New York: Wiley.

Freeman, K.C. (1970). On the Disks of Spiral and S0 Galaxies. The Astrophysical Journal 160: $811-830$

Hubble, E. P. (1930). Distribution of luminosity in elliptical nebulae. The Astrophysical Journal 71, 231-276.

Khosroshahi, H. G., Raychaudhury, S., Ponman, T.J., Miles, T.A., Forbes, D.A. (2004). Scaling relations in early-type galaxies belonging to groups. Monthly Notices of the Royal Astronomical Society, 349: 527-534.

Khosroshahi, H. G., Wadadekar, Y., Kembhavi, A., Mobasher, B. (2000a). Correlations among global photometric properties of disk galaxies. The Astrophysical Journal letters 531: 103106.

Khosroshahi, H. G., Wadadekar, Y., Kembhavi, A. (2000b). Correlations among global photometric properties of disk galaxies. The Astrophysical Journal 533: 162-171.

King, I. R. (1962). The structure of star clusters. I. An empirical density law, Astronomical Journal, Vol. 67, 471.

King, I R. (1966). The structure of star clusters. III. Some simple dynamical models. Astronomical Journal, Vol. 71, 64.

Kormendy, J. (1977). Brightness distributions in compact and normal galaxies. III - Decomposition of observed profiles into spheroid and disk components. The Astrophysical Journal, Vol. 217, 406-419.

Koza, J.R. (1992). Genetic Programming: on the programming of computers by means of natural selection. MIT Press.

Koza, J.R. (1994). Genetic Programming II: Automatic Discovery of Reusable Programs. MIT Press.

Koza, J.R. (1992). Genetic Programming: on the programming of computers by means of natural selection. MIT Press.

Koza, J.R. (1994). Genetic Programming II: Automatic Discovery of Reusable Programs.

Schombert, J. M. \& Bothun, G. D. (1987). The methodology and reliability of determining bulge-to-disk ratios for spiral galaxies. Astronomical Journal (ISSN 0004-6256), Vol. 93, 60-73.

Sersic, J. L. (1968) Atlas de Galaxies Australes Cordoba: Observatorio Astronomica

Sparke, L. S. \& Gallagher III, J. S. (2000). Galaxies in the Universe: An introduction. Cambridge University Press.

Van den Bergh, S. (1998) Galaxy Morphology and classification. Cambridge University Press.

Yao, X., (1996) An overview of evolutionary computation, Chinese J. Adv. Software Res., Vol. 3, No. 1, 12-29.

Yao, X, Liu, Y. and Lin, G. (1999) Evolutionary Programming Made Faster. In: IEEE Transaction on Evolutionary Computation Vol. 3, No. 2. 82-102.

Wadadekar, Y., Robbason, B., Kembhavi, A. (1999). Two-dimensional Galaxy Image Decomposition. Astronomical Journal, Vol 117, Issue 3, 1219-1228. 